Revue internationale P.M.E.

Économie et gestion de la petite et moyenne entreprise

Revue

internationale

PME

\title{
La PME et les processus de certification sociale SA 8000 Le cas de WebTel
}

\section{Manal El Abboubi et Annie Cornet}

Volume 20, numéro 3-4, 2007

URI : https://id.erudit.org/iderudit/1008525ar

DOI : https://doi.org/10.7202/1008525ar

Aller au sommaire du numéro

\section{Éditeur(s)}

Presses de l’Université du Québec

ISSN

0776-5436 (imprimé)

1918-9699 (numérique)

Découvrir la revue

\section{Citer cet article}

El Abboubi, M. \& Cornet, A. (2007). La PME et les processus de certification sociale SA 8000 : le cas de WebTel. Revue internationale P.M.E., 20(3-4), 95-119. https://doi.org/10.7202/1008525ar

\section{Résumé de l'article}

Les différentes recherches en cours sur la responsabilité sociale des entreprises (RSE) soulignent l'importance de s'intéresser aux PME et la nécessité de disposer d'études de cas approfondies afin d'éclairer les raisons pour lesquelles les PME s'engagent ou non dans une démarche de RSE et les processus associés à la volonté de se positionner dans ce champ de la RSE. Cet article présente une étude de cas d'une PME qui se trouve dans un secteur fortement décrié en matière de responsabilité sociale (Pichault et Zune, 2000), à savoir un centre d'appels. L'analyse porte sur le processus de labellisation SA 8000 qu'a suivi cette PME en portant une attention spéciale aux facteurs de contingence qui expliquent cette volonté de labellisation mais en faisant aussi une lecture politique du processus d'implication des parties prenantes, partenaires obligés de ce processus de labellisation. L'analyse s'appuie sur une grille contextualiste (Pettigrew, 1990, 1985) qui permet d'inclure l'interaction entre des facteurs de contingence (contexte interne et externe) et le jeu politique des acteurs qui façonnent le contenu du changement, en l'occurrence, le processus de labellisation. L'analyse montre la fragilité des PME dans un tel processus de certification et les difficultés liées à leur faible pouvoir pour impliquer les parties prenantes, nécessaires à la labellisation. Elle montre aussi le rôle clé que jouent les valeurs du dirigeant-propriétaire et les opportunités qui se créent autour de ce processus pour le personnel, demandeur d'une plus grande formalisation de la GRH. Finalement, le texte met en évidence l'intérêt d'études qualitatives et longitudinales pour comprendre les processus de RSE dans les PME.
Ce document est protégé par la loi sur le droit d'auteur. L'utilisation des services d’Érudit (y compris la reproduction) est assujettie à sa politique d'utilisation que vous pouvez consulter en ligne.

https://apropos.erudit.org/fr/usagers/politique-dutilisation/ 


\title{
La PME et les processus de certification sociale SA 8000 \\ Le cas de WebTel
}

Manal EL ABBOUBI

Annie CORNET

HEC École de gestion

Université de Liège

\section{MOTS CLÉS}

\section{Responsabilité sociale des entreprises - Certification sociale SA8000 - Contextualisme - Centre d'appels}

\begin{abstract}
LES AUTEURS
Manal El AbBoubl est chercheure doctorante à HEC École de gestion de l'Université de Liège à l'UER Management pour le service GRH et management des organisations. Adresse: HEC École de gestion de l'Université de Liège, UER Management, Service GRH et management des organisations, B31, boîte 48, Sart Tilman - 4000 Liège. Courriel: $<$ melabboubi@ulg.ac.be>.

ANNIE CORNET est professeure à HEC École de gestion de l'Université de Liège dans le département Management pour le service GRH et management des organisations, cours et formation en GRH, théorie des organisations et gestion de la diversité. Elle est la créatrice et responsable de l'Unité de recherche EgiD (Études sur le genre et la diversité en gestion); elle a mené plusieurs recherches sur l'entrepreneuriat féminin mais aussi sur les discriminations dans l'emploi. Adresse: HEC École de gestion de l'Université de Liège, Unité de recherche EgiD (Études sur le genre et la diversité en gestion), B31, boîte 48, Sart Tilman - 4000 Liège. Courriel: <annie.cornet@ulg.ac.be>.
\end{abstract}

\section{RÉSUMÉ}

Les différentes recherches en cours sur la responsabilité sociale des entreprises (RSE) soulignent l'importance de s'intéresser aux PME et la nécessité de disposer d'études de cas approfondies afin d'éclairer les raisons pour lesquelles les PME s'engagent ou non dans une démarche de RSE et les processus associés à la volonté de se positionner dans ce champ de la RSE. Cet article présente une étude de cas d'une PME qui se trouve dans un secteur fortement décrié en matière de 
responsabilité sociale (Pichault et Zune, 2000), à savoir un centre d'appels. L'analyse porte sur le processus de labellisation SA 8000 qu'a suivi cette PME en portant une attention spéciale aux facteurs de contingence qui expliquent cette volonté de labellisation mais en faisant aussi une lecture politique du processus d'implication des parties prenantes, partenaires obligés de ce processus de labellisation. L'analyse s'appuie sur une grille contextualiste (Pettigrew, 1990, 1985) qui permet d'inclure l'interaction entre des facteurs de contingence (contexte interne et externe) et le jeu politique des acteurs qui façonnent le contenu du changement, en l'occurrence, le processus de labellisation. L'analyse montre la fragilité des PME dans un tel processus de certification et les difficultés liées à leur faible pouvoir pour impliquer les parties prenantes, nécessaires à la labellisation. Elle montre aussi le rôle clé que jouent les valeurs du dirigeant-propriétaire et les opportunités qui se créent autour de ce processus pour le personnel, demandeur d'une plus grande formalisation de la GRH. Finalement, le texte met en évidence l'intérêt d'études qualitatives et longitudinales pour comprendre les processus de RSE dans les PME.

\begin{abstract}
The various existing researches on Corporate Social Responsibility (CSR) stress the importance to be interested in SMEs and the need for having in-depth case studies which allow to highlight the reasons for SMEs to enter (or not) into CSR and the processes associated with the will to position themselves in this field of CSR. This paper presents the case study of a SME in a sector strongly denigrated as regards social responsibility, namely a call center. The analysis focuses on the SA 8000 certification process followed by this SME, with a special attention on contingency factors explaining this will of certification, but also on a political reading of the process of stakeholders' involvement, necessary partners of this certification process. The analysis is based on a contextualist grid (Pettigrew, 1990, 1985) which allows including the interaction between contingency factors (internal and external context) and the political game of the actors who shape the contents of change that is here the certification process. The analysis shows the weakness of SMEs in such a process of certification and the difficulties related to their weak capacity to imply the necessary stakeholders for certification. It also shows the key role of the owner's values and the opportunities created around this process for staff, demanding a stronger formalisation of HRM. Finally, the paper highlights the interest of qualitative and longitudinal studies in order to understand CSR processes in SMEs.
\end{abstract}

\title{
RESUMEN
}

Las diferentes búsquedas sobre la responsabilidad social de las empresas (RSE) notan la importancia de interesarse a las PyMEs y la necesidad de disponer de estudios de casos detallados que permitan de aclarar las razones por cuales las PyMEs entran (o no entran) en la RSE y los procesos asociados a la voluntad de posicionarse en este campo de la RSE. Este artículo presenta un estudio de casos de una PME cual se encuentra en un sector muy controvertido con respecto a la responsabilidad social, un centro de llamadas. El análisis se concentra en el proceso de labelisación SA 8000 que esta PME siguió con enfoque sobre los factores de contingencia cuales explican esta voluntad de labelisacion pero también una lectura 
política del proceso de implicaciones de los partenarios necesarios para este proceso de labelisacion. La base del análisis es un cuadro contextualista (Pettigrew, 1990, 1985) cual permite de incluir la interacción entre factores de contingencia (contexto interno y externo) y el juego político de los actores quienes modelan el contenido del cambio, en otras palabras aquí, el proceso de labelisacion. El análisis muestra la debilidad de las PyMEs en este proceso de labelisacion y las dificultades vinculadas al bajo poder para implicar los partenarios necesarios para labelisacion. El análisis también muestra el papel clave que juegan los valores del dirigentepropietario y las oportunidades que se crean alrededor de este proceso para el personal, pidiendo una más grande formalización de la gestión de los recursos humanos. Finalmente, el texto pone en evidencia el interés de estudios cualitativos y longitudinales para entender los procesos de RSE en las PyMEs.

\section{ZUSAMMENFASSUNG}

Die verschiedenen laufenden Forschungsarbeiten über die soziale Verantwortung der Unternehmen (SVU) unterstreichen die Wichtigkeit, sich an der Rolle der KMU zu interessieren und über vertiefte Fallstudien zu verfügen, die über die Gründe aufzuklären vermögen, wieso sich KMU den SVU-Praktiken verschreiben. Der folgende Artikel präsentiert eine Fallstudie eines kleinen und mittleren Unternehmens, das sich in einer SVU-freien Branche befindet (Pichault und Zune, 2000) - nämlich ein Call-Center. Die Analyse untersucht den Zertifizierungsprozess (SA 8000) dieses Unternehmens mit einem Fokus auf die erklärenden Kontingenzfaktoren, die den Willen zur Zertifizierung erklären. Auch zur Analyse gehört eine unternehmenspolitische Betrachtung der involvierten Anspruchsgruppen, vor allem den im Zertifizierungsprozess verpflichteten Partnern. Methodisch basiert die Analyse auf einem kontextuellen Raster (Pettigrew, 1990, 1985), das den Einbezug der Interaktion zwischen den Kontingenzfaktoren (interner und externer Kontext) und politischen Zusammenspiel der Akteure, die den Inhalt der Veränderung bilden, in vorliegendem Fall der Zertifizierungsprozess. Die Analyse zeigt die Zerbrechlichkeit der KMUs in einem solchen Prozess und die relative Verhandlungsschwäche, um die Anspruchsgruppen in den Prozess einzubinden - eine notwendige Bedingung im Zertifizierungsprozess. Die Analyse zeigt aber auch auf, welche Schlüsselrolle die Werte des Geschäftsführers und die neuen Möglichkeiten, die sich für die Mitarbeitenden im Prozess ergeben sowie die Aufforderung für eine stärkere Formalisierung des Personalmanagements. Abschliessend hebt der Text die Wichtigkeit von qualitativen Studien mit Langzeitperspektive hervor, um das Verständnis der KMU im Prozess hin zu SVU-Praktiken zu verstehen.

\section{Introduction}

Cet article propose d'analyser la RSE dans les PME à partir d'une étude de cas d'un centre d'appels qui entame une opération de labellisation SA 8000. L'analyse oppose une vision rationaliste du processus à une vision qui intègre les facteurs de contingence et une lecture politique de l'implication des parties prenantes, partenaires obligés de ce projet de labellisation. L'analyse s'appuie sur une grille contextualiste (Pettigrew, 1985, 1990) qui permet d'inclure

Revue internationale P.M.E., vol. 20, n $\mathrm{n}^{\text {os }} 3-4,2007$ 
l'interaction entre des facteurs de contingence (contexte interne et externe) et le jeu politique des acteurs qui façonnent le contenu du changement, en l'occurrence le processus de labellisation. Nous présentons dans un premier temps la façon dont est posée la problématique de la RSE dans les PME, puis notre grille d'analyse (le contextualisme). S'en suit une brève présentation du cas. L'analyse présente dans un premier temps une vision rationaliste de la labellisation, enrichie par une analyse processuelle et longitudinale qui prend en compte des variables de contingence propres à la réalité des PME et le jeu politique des acteurs. Cela nous amène à montrer comment et pourquoi l'organisme de certification en arrive à assouplir ses exigences et à prendre en compte dans une certaine mesure les réalités de la PME sans pour autant remettre en cause la cohérence du modèle global.

\section{La RSE dans les PME}

La responsabilité sociale des entreprises se définit comme les pratiques mises en place par une organisation pour garantir la qualité de vie au travail, le respect des droits humains et syndicaux, une politique de gestion des ressources humaines juste et équitable. Elle se définit aussi comme un processus d'amélioration dans le cadre duquel les entreprises intègrent de manière volontaire, systématique et cohérente des considérations d'ordre social (people) (Allouche, 2005). Elle est une dimension d'un concept plus large, la responsabilité sociétale, qui englobe notamment la notion d'investissements responsables (profit) et de protection de l'environnement (planet) et s'articule aux démarches s'inscrivant dans le développement durable.

Il existe plusieurs labels ou certifications ciblant les actions menées en matière de RSE. Ces processus de certification et de normalisation induisent la formalisation des procédures de travail et des politiques de GRH (Chaouki et Yanat, 2004) avec un souci pour le volet social de la mise en conformité avec les droits fondamentaux repris dans les accords internationaux tels que la sécurité de travail, des horaires de travail décents, des rémunérations permettant de vivre au-dessus du seuil de pauvreté et l'existence d'une délégation syndicale (Igalens et Peretti, 2004). Les processus de labellisation ont été largement conçus pour les grandes entreprises et organisations (Business and Society, 2005) qui, d'une part, disposent de moyens financiers à investir dans l'obtention de ces labels et, d'autre part, ont des politiques de gestion formalisées.

Les PME ont certaines caractéristiques internes qui nécessitent une analyse différenciée de l'implantation des processus de certification de la RSE. Elles se caractérisent par un poids important du dirigeant-propriétaire dans la gestion de l'entreprise mais aussi par des politiques de gestion des res- 
sources humaines souvent informelles (Mahé de Boislandelle, 1998; Bayad et Nebenhaus, 1998; Bayad et al., 1995), qualifiées parfois d'arbitraires (Pichault et Nizet, 2000). Les modes d'organisation du travail sont basés généralement sur la polyvalence (division horizontale faible) et un fort contrôle hiérarchique (division verticale forte), avec la supervision directe comme principal mode de coordination. Au plan stratégique, les PME sont dans une forte dépendance à leur environnement et à certains acteurs dont les donneurs d'ordres qui, de plus en plus, fixent les règles du jeu (ORSE, 2005; Laval, 1998) et influencent les pratiques de ces PME.

Une étude de l'Union européenne vise à établir les différentes formes que peut prendre la RSE dans les PME telles que l'implication dans les communautés locales, l'investissement dans le personnel par des actions de formation, des possibilités d'aménagement du temps de travail, des améliorations de la santé-sécurité au travail, l'intégration de publics fragilisés ou marginalisés, le maintien à l'emploi et/ou le recrutement de travailleurs âgés ou l'implication dans des projets de coopération internationale (Union européenne, 2006).

Peu de labels et de certification s'adaptent à ces réalités des PME. Les différentes caractéristiques des PME, telles que leur taille et le peu de moyens et ressources qu'elles peuvent investir dans un tel processus, constituent un frein au processus de labellisation (Berger-Douce, 2004; Quairel et Auberger, 2005). Plusieurs d'entre elles se voient obligées de s'inscrire dans une telle démarche pour répondre aux exigences de leurs donneurs d'ordres (Laval, 1998), eux-mêmes impliqués dans un processus de labellisation avec l'obligation de conformité pour leurs parties prenantes. Au-delà de cette action réactive, certaines PME, souvent sous l'influence des valeurs du dirigeant-propriétaire (Murillo et Lozano, 2006; Union européenne, 2006; Dupuis, Haned et Le Bas, 2006), optent pour des stratégies proactives qui vont au-delà d'une mise en conformité. C'est à ce type de PME que nous allons nous intéresser. En effet, nous allons décrire et analyser le processus d'une PME qui s'engage dans un processus de RSE avec le label SA 8000.

\section{Le label SA 8000}

La norme SA $8000^{1}$ porte sur la qualité sociale de l'entreprise qui en bénéficie. La norme SA 8000 repose sur le respect de la Déclaration des droits de l'homme, le Pacte des droits économiques et sociaux, les Conventions de base de l'Organisation internationale du travail (OIT) et la Convention de New York sur les droits de l'enfant. Ce label garantit que l'entreprise

1. <http://www.sa-intl.org/ http://www.sa-intl.org >. 
respecte un certain nombre de normes internationales en matière de travail, à savoir cinq principes de base que sont la liberté syndicale et de négociation collective, l'absence de travail des enfants, l'absence de discrimination et de travail forcé auxquels s'ajoutent les conventions internationales relatives à la santé et à la sécurité des travailleurs et le contrôle du temps de travail et des heures supplémentaires. La norme SA 8000 est intégrée au système de management et prévoit des améliorations progressives. La vérification est effectuée par des experts indépendants accrédités par la Social Accountability International (SAI). Elle suppose l'implication de certaines parties prenantes internes (employés essentiellement) et externes (fournisseurs et partenaires d'affaires). L'adoption de la SA 8000 se traduit également par un rapport porté à la connaissance du public dont les principales caractéristiques sont présentées sur le site Internet de la SAI. Il est généralement publié tous les ans et vérifié par la SAI. La certification SA 8000 et le programme d'implication de l'entreprise doivent aider le consommateur et l'investisseur à repérer les entreprises qui respectent les droits de l'homme sur les lieux de travail.

Les processus de certification et de labellisation proposent un modèle normatif, très formalisé. Ce modèle est basé sur:

- le diagnostic de la situation initiale, soit l'ensemble des caractéristiques que possède l'organisation avant son engagement dans le processus de certification. Au terme du diagnostic, il s'agit d'évaluer le degré de conformité à $\mathrm{T}^{\mathrm{o}}$ de l'organisation à un ou plusieurs critères demandés par le label SA 8000 (huit conventions collectives du droit international du travail);

- la définition des résultats escomptés et objectifs à atteindre, en l'occurrence la conformité aux critères exigés pour l'obtention du label et la certification de cette conformité par des certificateurs accrédités par la SAI;

- les actions à mener pour atteindre les résultats escomptés (ajustement des modes d'organisation du travail et des politiques de GRH et formalisation dans des procédures, adhésion des parties prenantes) et les moyens financiers et humains qui seront affectés à l'atteinte de cet objectif;

- la certification qui confirme la conformité en regard des critères retenus par le label et valide un plan d'action pour les points nécessitant encore des améliorations. 
Le processus de certification s'inscrit donc dans un processus de changement qui vise, en fonction du diagnostic, à adapter les procédures de travail et à élaborer un plan d'action pour se mettre en conformité avec les exigences de la certification.

\section{Figure 1}

\section{Critères de certification sociale $\mathrm{SA} 8000$ Le processus de certification}

\begin{tabular}{|c|c|c|}
\hline $\begin{array}{l}\text { Diagnostic } \\
\text { de la situation initiale }\end{array}$ & & $\begin{array}{l}\text { Certification par } \\
\text { des auditeurs } \\
\text { agréés SA } 8000\end{array}$ \\
\hline $\begin{array}{l}\text { Travail des enfants } \\
\text { Travail forcé } \\
\text { Hygiène et sécurité } \\
\text { Liberté syndicale et droit } \\
\text { à la négociation collective } \\
\text { Discrimination } \\
\text { Pratiques disciplinaires } \\
\text { Temps de travail } \\
\text { Rémunération } \\
\text { Système de management }\end{array}$ & $\begin{array}{l}\text { Adaptation et rédaction } \\
\text { des procédures de travail. } \\
\text { Élaboration d'un plan } \\
\text { d'action. }\end{array}$ & $\begin{array}{l}\text { Travail des enfants } \\
\text { Travail forcé } \\
\text { Hygiène et sécurité } \\
\text { Liberté syndicale } \\
\text { et droit à la } \\
\text { négociation collective } \\
\text { Discrimination } \\
\text { Pratiques disciplinaires } \\
\text { Temps de travail } \\
\text { Rémunération } \\
\text { Système de management }\end{array}$ \\
\hline$\underbrace{\underbrace{\prime}}$ & & $\underbrace{\underbrace{2}}$ \\
\hline $\begin{array}{l}\text { Un ou plusieurs éléments } \\
\text { ne sont pas respectés. }\end{array}$ & & $\begin{array}{l}\text { Tous les éléments sont } \\
\text { respectés. }\end{array}$ \\
\hline
\end{tabular}

Le processus s'inscrit totalement dans un plan d'action rationaliste, piloté par l'équipe dirigeante, avec l'aide d'analystes et de consultants, qui suit un certain nombre d'étapes prédéterminées telles qu'elles sont schématisées ci-dessous. 


\section{FiguRE 2}

Les étapes formelles du processus de certification SA 8000

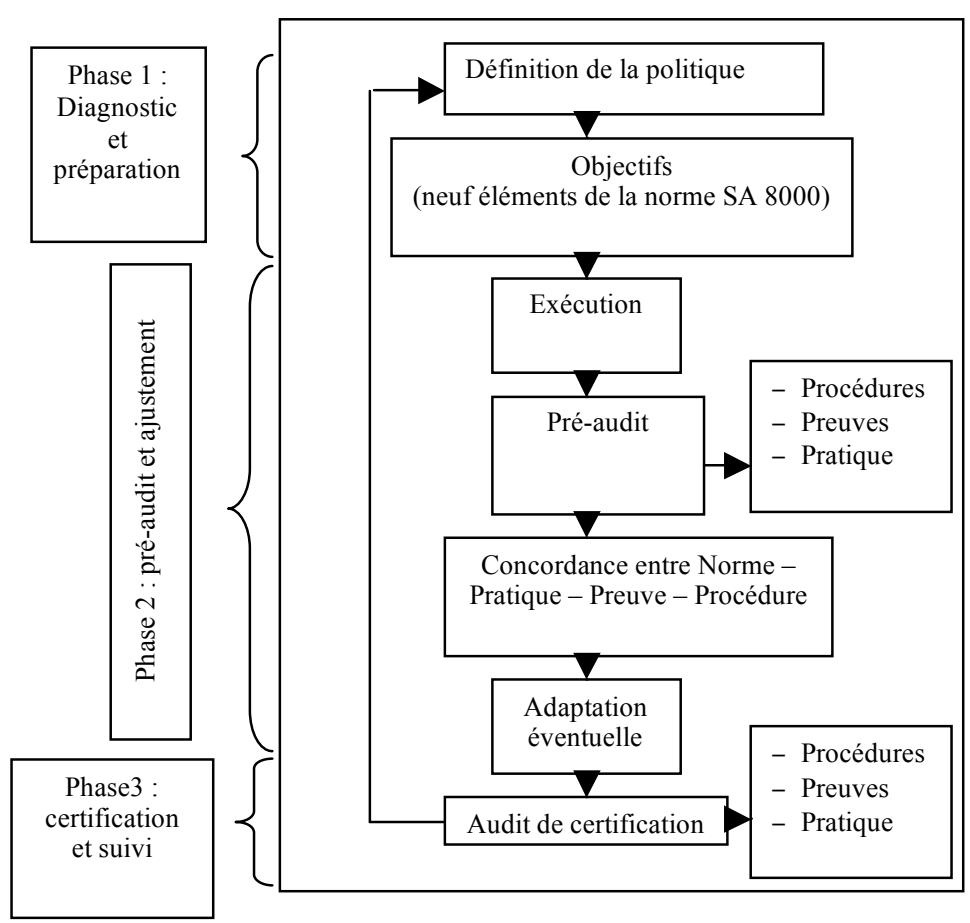

Le processus de certification s'appuie sur une analyse en cascade structurée autour des objectifs de la norme SA 8000. Nous ne nous arrêterons pas sur la définition de tous les éléments de ce processus, mais nous marquons un arrêt sur les particularités qui caractérisent les étapes d'audit, à savoir les 3P que sont les Procédures, les Preuves, les Pratiques.

Procédures. L'audit exige que chaque action soit formalisée par un écrit dont la traçabilité puisse être vérifiable. Les procédures écrites sont les premières sources d'information et d'évaluation pour les auditeurs SA 8000.

Preuves. Les certificateurs ont la charge de collecter les preuves des pratiques engagées par l'organisation. Il incombe à l'organisation de préparer les preuves de ses engagements. Celles-ci peuvent être écrites (documents officiels) ou orales (entrevues lors des audits). 
Pratiques réelles sur le terrain. Elles sont validées lors de l'observation menée par les auditeurs et par des interviews avec différents acteurs tels que les délégués syndicaux, un échantillon de membres du personnel, des parties prenantes.

Dans la logique de ce modèle rationaliste, proche de la planification stratégique, le manager dispose d'outils d'évaluation qui doivent l'aider à surveiller le bon accomplissement du processus SA 8000 (c'est le principe des audits internes et externes) et à réviser en conséquence ses objectifs, les actions menées et les ressources allouées.

Comme le soulignent Igalens et Peretti, les outils d'évaluation permettent «de vérifier qu'une entreprise dit ce qu'elle fait et fait ce qu'elle dit, qu'elle le fait dans les règles de l'art et qu'elle maîtrise les risques qui pèsent sur elle» (Igalens et Peretti, 2004, p. 241). On se situe clairement dans un modèle de gestion normatif et rationaliste (Brouwers, Cornet et Gutierrez, 1997). Ce modèle postule que le pôle de décision est unique (dans une PME,le gestionnaire-propriétaire) et que ce gestionnaire pilote le processus. Dans une telle perspective, la formation des stratégies (ici la labellisation) est conçue comme un processus séquentiel dans lequel le décideur a pleine maîtrise, le suivi de cette dernière étant assuré par des outils d'évaluation. Si des problèmes surgissent (résistances au changement, par exemple), c'est que l'analyse préalable des solutions possibles n'a pas été menée adéquatement ou que le plan d'action n'a pas suffisamment impliqué les parties prenantes.

Cette analyse du processus de labellisation qui se limite à la description de ces étapes, des outils mis en œuvre et des moyens affectés à cette politique rate des aspects importants qui permettent de mieux comprendre le processus, les enjeux et l'importance de facteurs de contexte. C'est ce que nous allons tenter de démontrer en menant une analyse contextualiste de la mise en place de la RSE dans un contexte de PME à partir de l'analyse d'un processus de certification SA 8000 dans un centre d'appels.

\section{L'approche contextualiste}

L'approche contextualiste de Pettigrew $(1990,1985)$ propose une grille d'analyse multidimensionnelle et longitudinale des processus de changement. Trois niveaux d'analyse sont proposés: le contenu, le contexte et le processus. Il s'agit de montrer comment les acteurs impliqués dans le processus de changement s'emparent du contexte (contraintes et opportunités) pour influencer le processus de changement (contenu) dans un sens qui leur permet de préserver leurs intérêts stratégiques (Crozier et Friedberg, 1977). 
Le contenu renvoie à l'objet même du changement. Il s'agira, ici, des dimensions de l'organisation affectées par le processus de labellisation SA 8000: stratégie et relations avec les partenaires d'affaires, modes d'organisation du travail et politiques de gestion des ressources humaines.

Le contexte renvoie aux éléments interne et externe qui vont influencer le processus de changement mais aussi être mobilisés et construits comme contraintes et potentialités par les acteurs. Le contexte interne analyse l'interaction avec la structure organisationnelle et les modes d'organisation $\mathrm{du}$ travail, la culture organisationnelle, le mode de direction et de prise de décision, les politiques de gestion des ressources humaines mais aussi les moyens financiers et les infrastructures techniques et immobilières. Le contexte externe s'intéresse aux effets sur le processus de changement de l'environnement social et culturel (normes et valeurs), économique, politique, légal, technologique, voire géographique.

Le processus introduit la dimension temporelle et vise à analyser comment et pourquoi le contenu du changement évolue selon le positionnement politique des acteurs, leurs enjeux et stratégies.

L'analyse contextualiste peut mobiliser dans l'analyse du processus différents modèles théoriques (Brouwers, Cornet et Gutierrez, 1997), nous en retiendrons deux: le modèle contingent et le modèle politique tel qu'il est schématisé ci-dessous.

Figure 3

\section{Grille d'analyse contextualiste}

\begin{tabular}{|c|c|c|c|}
\hline $\begin{array}{l}\text { Le modèle de la } \\
\text { contingence }\end{array}$ & EXTERNE & \multicolumn{2}{|c|}{$\begin{array}{l}\text { Environnement socioculturel, } \\
\text { économique, politique, légal, } \\
\text { technologique, géographique. }\end{array}$} \\
\hline $\begin{array}{c}\text { CONTENU } \\
\text { Stratégie } \\
\text { Organisation du } \\
\text { travail }\end{array}$ & INTERNE & $\begin{array}{l}\text { Stratégie } \\
\text { organisat } \\
\text { GRH, m } \\
\text { infrastru } \\
\text { immobil }\end{array}$ & $\begin{array}{l}\text { lture } \\
\text { de décision, }\end{array}$ \\
\hline Politiques de GRH & $\begin{array}{l}\text { PROCESSUS DE LABELL } \\
\text { Actions, réactions et interacti } \\
\text { différentes parties concernées }\end{array}$ & $\begin{array}{l}\text { ATION } \\
\text { is entre }\end{array}$ & $\begin{array}{l}\text { Modèle } \\
\text { politique }\end{array}$ \\
\hline
\end{tabular}


Les idées maîtresses de l'approche de la contingence sont les suivantes (Burns et Stalker, 1961; Lawrence et Lorsch, 1967; Miles et Snow, 1978; Mintzberg, 1982):

- Les organisations sont des systèmes ouverts qui ont besoin d'être soigneusement administrés si l'on veut répondre aux besoins internes, les équilibrer et les adapter à l'environnement et à ses modifications.

- Il n'y a pas une seule façon d'organiser le travail; tout dépend du type de tâche ou du type d'environnement auquel on a affaire.

- Les dirigeants doivent avant tout arriver à de bons ajustements. Diverses méthodes au sein d'une seule organisation peuvent s'avérer nécessaires pour effectuer des tâches différentes. Les divers types d'organisation correspondent à des environnements différents.

En d'autres termes, l'essentiel de la théorie de la contingence revient à considérer qu'organiser impose de faire des choix et qu'une organisation ne peut être efficace que dans la mesure où sont rendus compatibles la stratégie, la structure, les techniques, les besoins des membres et l'environnement. La cohérence ( fit) entre les diverses composantes organisationnelles et le contexte dans lequel œuvre l'organisation constitue le premier facteur déterminant le succès. Cela s'inscrit totalement dans la conclusion de Quairel et Auberger (2005, p. 23) qui soutiennent que: «La mise en place d'un processus de RSE dans les PME suppose une cohérence entre les représentations de l'entrepreneur, les ressources et les outils dont il dispose et les pressions de l'environnement.»

L'approche politique (Crozier et Friedberg, 1977; Pfeffer, 1981) s'exprime en termes de rapports de force, de défense d'intérêts, de contrôle de zones d'incertitude. Elle introduit l'importante notion d'acteur, ignorée par le schéma rationnel qui ne considère comme décideur légitime que l'équipe dirigeante et les spécialistes qui l'assistent. L'acteur est ici défini comme le membre d'un groupe d'intérêt, capable d'exercer une influence sur d'autres acteurs et de déployer en conséquence des stratégies appropriées (défensives ou offensives). Une telle conception a évidemment des implications importantes sur la manière d'analyser le processus de labellisation. Au lieu de considérer cette dernière comme un processus séquentiel, «objectif» et structuré, le modèle politique met en évidence les influences susceptibles d'être exercées à chacune des étapes de ce processus. Les enjeux du contrôle varient en fonction des atouts dont disposent les différents partenaires dans la structure formelle de l'organisation: ces derniers ne mobiliseront leurs ressources et ne déploieront leurs stratégies que si, à leurs yeux, les enjeux d'une telle mobilisation en valent la peine. Une partie de la littérature sur 
l'implication des parties prenantes introduit cette dimension politique dans l'analyse d'un processus de RSE (Freeman, 1994; Mitchell, Agle et Wood, 1997). L'analyse politique aboutit à une conception très particulière des processus de changement (Crozier et Friedberg, 1977, p. 325-347) et des enjeux de la RSE (Aggeri et al., 2005) qui sera mobilisée dans notre analyse:

- le changement est systémique, c'est-à-dire contingent au système d'action concret qui l'élabore et auquel il s'applique;

- pour qu'il y ait changement, il faut que le système d'action se transforme lui-même et qu'il y ait apprentissage collectif de nouveaux modèles de relations entre acteurs, de nouvelles règles du jeu. Comme le soulignent Aggeri et al. (2005, p. 247), les acteurs ne se contentent pas de figures imposées (le modèle tel qu'il est défini et précisé dans l'accompagnement du label) mais «ils pratiquent également des figures libres».

Nous allons voir que cet apprentissage collectif de nouveaux modèles de relation va prendre tout son sens dans l'implication du personnel dans le processus de labellisation mais aussi dans les relations avec les différentes parties prenantes de l'entreprise (partenaires et fournisseurs notamment), ensemble fragmenté, constitué de relations multiples et hétérogènes (Aggeri et al., 2005). Comme le signalent ces auteurs, «un référentiel n'est jamais neutre. Il n'indique ni le vrai, ni le juste. C'est une convention qui est le résultat d'un compromis entre différentes stratégies d'acteurs » (Aggeri et al., 2005, p. 260).

\section{Présentation du cas WebTel}

WebTel (nom fictif) est une entreprise familiale lancée par les frères Grégoire (nom fictif) en 2001. C'est un centre d'appels qui fait affaire dans le télémarketing. Créé depuis 2000, le siège social est à Paris alors que le site d'exploitation premier est en Wallonie (Belgique). L'activité principale est l'émission et la réception d'appels pour des opérations téléphoniques de vente, de sondage, de prise de rendez-vous et de classification de dossier. En janvier 2004, WebTel, pour réduire ses coûts de production, a commencé à sous-traiter une partie de sa production francophone à un centre d'appels au Maroc. En 2005, elle a ouvert un nouveau site d'exploitation en Flandre (Belgique). Le nouveau site se compose uniquement de plateformes de production:émission et réception d'appels. Tous les autres services restent centralisés en Wallonie. En moyenne, 20 agents travaillent sur chacun des sites. Les contrats de travail sont majoritairement des contrats à durée déterminée. Les clients de WebTel (donneurs d'ordres) sont des grandes entreprises. 
Pendant le lancement de l'entreprise en 2001, WebTel a réalisé un chiffre d'affaires de $120000 €$. Depuis cette date, l'entreprise n'a cessé de progresser avec un chiffre d'affaires de $1000000 €$ en 2005. En 2006, la répartition du chiffre d'affaires selon les différents sites de production a été la suivante:20\% réalisé depuis le sous-traitant au Maroc, 35 \% réalisé depuis le site principal de Wallonie, 30 \% réalisé depuis la succursale de Flandre, 15 \% réalisé depuis les autres antennes des sous-traitances ponctuelles (la Pologne, l'Espagne et la France). Par ailleurs, pour ce qui est de la répartition du chiffre d'affaires sur les marchés ciblés par les projets WebTel, elle se représente comme suit: $10 \%$ sur la Wallonie, 30 \% sur la Flandre, 50 \% sur la France, 5 \% sur la Suisse, $5 \%$ sur autre destination (Allemagne, Pays-Bas, Royaume-Uni).

La structure de WebTel est typiquement pyramidale. Le sommet est représenté par le directeur général. Se déclinent ensuite trois services: production, informatique et personnel. En janvier 2006, un service commercial a été créé, il sera dirigé par un des frères du DG. Le service informatique est géré par le frère du directeur général, qui est également associé. Le service du personnel est géré par l'épouse du DG, elle aussi associée. On est donc clairement dans la configuration d'une entreprise familiale. Le service de production est géré par la responsable de production. Elle a été l'intermédiaire officielle entre la direction et l'ensemble des agents opérateurs pour tous les sites de production à l'exception du sous-traitant marocain pour lequel la production est suivie directement par une superviseure qui assure la formation, la mise en place, le lancement des opérations et le traitement des données. Chaque entité est suivie par un superviseur qui assure la gestion de la production, le suivi des opérations quotidiennes et une partie de la gestion opérationnelle du personnel. À côté de leurs fonctions d'encadrement, les superviseurs ont les mêmes exigences de productivité téléphonique que les agents opérateurs, d'où la complexité de leur fonction. L'entreprise engage un personnel moyennement qualifié. Les représentants de la direction sont les seuls universitaires. Le personnel engagé est très souvent jeune (entre 16 et 30 ans). Les sites de production ne dépassent pas 25 postes de travail (pour les agents opérateurs). La politique de GRH est peu formalisée, la faible taille du centre d'appels et son implantation régionale permettent une ambiance de travail amicale qui contribue à la fidélisation des téléopérateurs qui connaissent les conditions de travail plus contraignantes des plus grands centres d'appels de la région.

WebTel dispose des nouvelles technologies en télécommunication à travers la location de lignes téléphoniques digitales. La grande fiabilité et la disponibilité à l'échelle internationale de celles-ci garantissent une gestion personnalisée du réseau de connexion. Par ailleurs, le gain sur les coûts est nettement meilleur par rapport aux lignes analogiques. À côté de cela, un 
nouveau logiciel de traitement des données est mis en place depuis 2005; c'est un serveur central qui permet la supervision instantanée de la production à distance. Cette technologie permet une flexibilité et une mobilité des superviseurs entre les sites de production tout en garantissant le suivi de leurs opérations et leurs équipes à distance. Parallèlement à cela, cette technologie répond aux exigences des donneurs d'ordres en matière de fluidité de la communication, de traitement et d'échange de l'information en temps réel. Par ailleurs, toute la gestion des bases de données relatives au traitement informatique est centralisée sur le site d'exploitation wallon.

En 2005, WebTel décide d'obtenir une certification sociale SA 8000 (Social Accountability 8000) pour son siège d'exploitation en Wallonie et sa succursale en Flandre. La certification SA 8000 est reconnue comme un des axes stratégiques des années 2005-2006. Un budget de plus de $30000 €$ est alloué. WebTel a su dépasser le cap des audits externes de certification officielle pour devenir en mai 2006 le premier centre d'appels européen à obtenir le label social SA 8000 . WebTel est à présent certifié «entreprise responsable socialement», et ce, jusqu'en 2008. Entre-temps, elle se trouve dans l'obligation de prouver des avancées stratégiques dans sa politique de gestion des ressources humaines et de suivi de ses relations avec ses parties prenantes, notamment les fournisseurs et les sous-traitants pour pouvoir prolonger sa certification.

\section{Méthodologie de la recherche}

Cette article est réalisé à partir d'une analyse qualitative, processuelle et longitudinale d'une étude de cas : un centre d'appels dans lequel nous avons suivi tout le processus de labellisation (SA 8000). La collecte des données a été réalisée grâce aux trois sources suivantes:

- la première source de données est l'observation pendant la période d'accompagnement de la PME lors de ses démarches de préparation de son dossier de certification sociale. Cette mission a duré plus de 18 mois. Elle a permis l'observation des comportements au moment où ils se sont présentés et leur compréhension. Par ailleurs, l'observation permet de détecter l'organisation informelle de travail, les pratiques indirectes et de mieux comprendre le contexte de travail;

- nous avons effectué des interviews durant lesquelles les opérateurs nous ont fait part de leurs opinions sur plusieurs aspects de la vie au sein de la PME touchant essentiellement la responsabilité sociale de 
l'entreprise. Ces opinions ont été croisées avec les données recueillies par les interviews avec la direction pour avoir une vue globale de la situation de l'entreprise;

- les sources documentaires analysées étaient les enquêtes de satisfaction du personnel, les manuels de procédures, les notes internes, les correspondances avec les parties prenantes externes (particulièrement les sous-traitants et les fournisseurs), les procédures de qualité et de sécurité. Ces documents ont aidé à contextualiser les données et les événements.

- l'analyse des données a donné lieu à un positionnement des actions posées par les acteurs sur une ligne du temps processuelle dans l'idée de déterminer quels sont les facteurs et les acteurs qui vont influencer l'évolution du processus de labellisation.

\section{Analyse contextualiste du processus de labellisation}

La période de certification que nous étudions s'étale de janvier 2005 à septembre 2006. Pour pouvoir mener une analyse longitudinale, nous avons découpé le processus en trois phases:

- Phase 1. Décision d'obtention du label et diagnostic (de janvier 2005 à juin 2005): elle correspond à la phase du diagnostic en regard des exigences de la norme SA 8000.

- Phase 2. Pré-audit et ajustement (de juillet 2005 à décembre 2005): elle concerne la période de lancement des audits internes et externes pour l'évaluation des pratiques et des procédures de mise en place. Des ajustements nécessaires à la certification sont alors prévus et mis en place pour la prochaine phase.

- Phase 3. Dernière étape avant la certification - certification - suivi (de janvier 2006 à septembre 2006): un audit externe concernant les procédures, les preuves et les pratiques est prévu pour les neuf éléments de la norme SA 8000 . Il s'en suit des plans d'action pour répondre aux non-conformités éventuelles relevées lors de l'audit de certification.

Nous allons reprendre chacune des phases en tentant d'identifier les éléments de contexte interne et externe qui façonnent le processus et qui sont mobilisés par les acteurs à travers les jeux politiques visant à augmenter ou à préserver leurs zones d'influence et leur pouvoir dans l'organisation. 


\section{Phase 1. Décision d'obtention du label et diagnostic (de janvier 2005 à juin 2005)}

La décision de la certification sociale SA 8000 est une réponse à des éléments du contexte externe et interne, très typique des PME:

- au regard du contexte externe, WebTel est confronté à des clients (donneurs d'ordres) de plus en plus exigeants quant à la production de garantie du respect des droits sociaux des travailleurs dans les différents sites et, plus particulièrement, chez le sous-traitant au Maroc;

- au regard du contexte interne, il y avait plusieurs revendications du personnel pour une formalisation plus grande des responsabilités et du partage des tâches et des politiques de gestion des ressources humaines, notamment en termes de gestion du temps de travail, type de contrat, possibilité de carrière. Comme dans beaucoup de PME, le personnel avait le sentiment que les décisions prises étaient arbitraires.

Ce label est initialement conçu pour des grandes entreprises. Obtenir la certification pour une PME constituait un véritable défi compte tenu de l'ampleur des exigences de ce label et des contraintes budgétaires. Malgré ces difficultés, la motivation de la direction de WebTel pour mener à terme le projet n'a fait que grandir. «Nous sommes convaincus que malgré notre petite taille, nous pouvons être une entreprise citoyenne et avoir des actions responsables socialement, et la certification SA 8000 en est un moyen » (directeur général de WebTel). Notre centre universitaire, spécialisé en changement organisationnel, est associé à la démarche pour une mission de conseil. Le directeur général considère qu'avec ce label il pourra simplifier les relations avec les donneurs d'ordres et augmenter le contrôle sur les employés à travers la rédaction des procédures exigées par le label. Le processus de certification sociale est engagé dans un système de distribution de pouvoir qui peut être qualifié de «centripète» (Pichault, 1993), avec une centralisation du pouvoir aux mains du directeur général, ce qui est caractéristique d'une PME. Celui-ci s'appuie sur sa ligne hiérarchique, en l'occurrence la responsable production, qui voit le processus de labellisation comme une menace à sa zone d'autonomie dans l'organisation. En effet, cette responsable était l'intermédiaire officiel entre la direction et les employés mais aussi l'interlocutrice privilégiée des parties prenantes externes (clients, fournisseurs, donneurs d'ordres). Cette responsable était un acteur clé de la GRH puisque la plupart des décisions du directeur général étaient basées sur l'avis de celle-ci, notamment les sanctions et les récompenses. Elle tire un grand pouvoir de la non-formalisation de la GRH et de son contrôle de la circulation de l'information entre les différentes parties en présence. Le personnel ne perçoit pas tout de suite l'intérêt que pourrait avoir le processus de labellisation au regard de ces préoccupations. 
Il souhaite plus d'objectivation dans la GRH, plus de transparence dans les critères d'évaluation et une meilleure prise en compte des facteurs de contexte, au-delà de la productivité immédiate, évaluée par une série d'indicateurs quantitatifs mobilisés par le DG pour évaluer la productivité des travailleurs.

En février 2005, pour réaliser la phase de diagnostic précédant l'audit, le centre universitaire partenaire du processus de labellisation suggère la réalisation d'une enquête de satisfaction auprès du personnel. Un questionnaire est envoyé à l'ensemble du personnel. Cette étude confirme l'insatisfaction du personnel au regard des politiques de GRH (gestion du temps de travail, possibilités de carrière, critères d'évaluation du personnel, gestion des contrats de travail, modalités d'octroi des primes et rémunérations, équité des politiques entre le site wallon et le site flamand).

Pour répondre aux exigences de la norme SA 8000, une charte éthique est rédigée qui reprend un engagement moral envers les huit domaines d'application de la norme. Cette charte est envoyée aux donneurs d'ordres, aux fournisseurs et au sous-traitant marocain, en leur demandant d'y adhérer. L'ensemble des parties prenantes confirme son adhésion aux huit principes déclinés dans ce document.

À la fin de cette première phase, le DG désigne un membre du personnel (une superviseuse) comme responsable certification. Son rôle sera la formalisation des procédures de travail en cohérence avec les exigences de la norme SA 8000. Il est prévu qu'elle consacre une partie de son temps de travail à cette tâche. Ce nouveau rôle la positionne comme un des interlocuteurs directs du DG pour différents points relatifs à l'organisation du travail, qui auparavant relevait exclusivement de la zone de pouvoir de la responsable production.

\section{Phase 2. Pré-audit et ajustement (de juillet 2005 à décembre 2005)}

Elle concerne la période de lancement des audits internes et externes pour l'évaluation des pratiques et des procédures mises en place. Il s'agit de vérifier si l'organisation couvre l'ensemble des exigences du label. La nomination de la responsable certification en juin 2005 a contribué à une distribution du pouvoir plus centrifuge. Cette nouvelle configuration a facilité les ajustements élémentaires de la GRH par rapport aux exigences normatives du SA 8000. En effet, cette responsable connaît bien les revendications du personnel. Elle continue d'ailleurs pour une partie de son temps de travail à assumer les tâches habituelles d'un opérateur du centre d'appels ainsi que les fonctions de superviseur. En novembre 2005, un audit interne est réalisé par le centre de recherche; son objectif est de pointer les décalages éventuels qui subsistent 
entre les pratiques et les exigences du label SA 8000. Cet audit est réalisé à partir d'analyse de documents et d'interviews des membres du personnel. Il met en évidence l'importance de mettre en place une réelle politique de gestion des ressources humaines, à savoir une politique de communication et de consultation du personnel, des perspectives de carrières pour les téléopérateurs et donc l'importance de valoriser les compétences acquises, la prise en compte d'éléments qualitatifs dans l'évaluation du personnel afin d'éviter que la productivité ne soit évaluée uniquement autour de critères quantitatifs. La démarche de formalisation de la GRH est en cours. Elle se traduit par l'implantation d'une structure de concertation avec le personnel avec la création du poste de délégué du personnel (vu la petite taille de l'entreprise, la représentation syndicale n'est pas obligatoire) et l'organisation de réunions mensuelles entre personnel et direction pour débattre des différents sujets relatifs à l'organisation du travail mais aussi aux perspectives de développement stratégique de l'entreprise. La responsable certification rédige des procédures internes, notamment pour la gestion des congés et des absences, les conditions d'octroi de primes, les modalités d'évaluation du personnel. La responsable de la production fait plusieurs tentatives pour tenter de bloquer ou de retarder le processus de formalisation et de mise en œuvre. En effet, ces procédures réduisent son pouvoir discrétionnaire et mettent en place de nouveaux canaux de communication reliant notamment la direction et la responsable certification et le délégué de personnel. Le schéma ci-dessous illustre les nouveaux canaux de communication qui se mettent en place et la nouvelle distribution interne du pouvoir:

\section{Figure 4}

\section{Distribution du pouvoir dans la phase 1 et 2 de la labellisation}

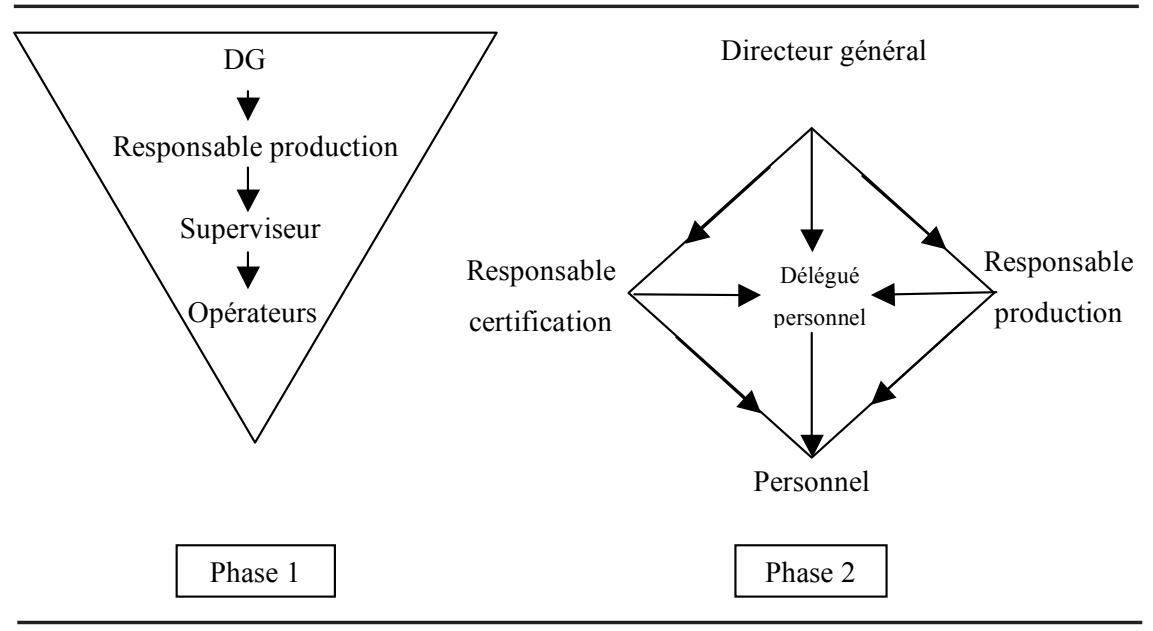


Au regard du contexte externe, en plus de l'engagement symbolique à travers la charte éthique, l'entreprise se voit dans l'obligation de demander à ces parties prenantes externes un engagement sur la conformité de leurs pratiques de gestion avec les huit domaines de la norme et l'acceptation de recevoir un auditeur externe pour certifier ces pratiques. La petite taille de l'entreprise ne lui accorde pas le poids voulu pour négocier confortablement la mobilisation de ses parties prenantes dans cette étape du processus de certification. En effet, certaines parties prenantes, qui avaient accepté de signer la charte éthique, refusent de s'engager sur leurs pratiques et la visite de l'auditeur externe. C'est le cas notamment de la société de nettoyage des locaux qui est une grande entreprise de nettoyage industriel. La stratégie de WebTel sera alors une stratégie de justification vis-à-vis des auditeurs du label qui vise à mettre en évidence le fait que sa spécificité comme PME l'empêche d'exiger de toutes ses parties prenantes (surtout les grandes entreprises pour lesquelles WebTel n'est finalement qu'un «petit» client) de se conformer aux exigences du label social. L'argument mobilisé dans le processus d'audit sera la taille de l'entreprise qui entraîne sa forte dépendance à l'égard de certains partenaires d'affaires et son faible pouvoir d'exigence quant à des ajustements dans l'organisation du travail notamment des grandes entreprises (société de nettoyage, opérateur de télécoms, producteur de logiciel bureautique, etc.). Des analyses ultérieures pourraient être réalisées afin d'essayer de comprendre le positionnement des différentes parties prenantes et leur degré de mobilisation au regard des typologies existantes (Mitchell, Agle et Wood, 1997; Sobzack et Girard, 2006) mais cela dépasse largement l'objet de cet article.

Il est intéressant de souligner qu'à cette phase le sous-traitant marocain accepte l'audit externe qui vise à poser un diagnostic sur les pratiques de gestion au regard des exigences de la norme qui dépassent les exigences des lois sociales marocaines. À ce stade, WebTel arrive donc à convaincre son sous-traitant de l'intérêt conjoint de la démarche.

\section{Phase 3. Dernières étapes avant la certification, certification et suivi (de janvier 2006 à avril 2006)}

Cette phase vise à assurer la conformité de l'organisation aux exigences du label à la suite des audits internes. La préparation de l'audit de suivi et des plans d'action démarre pour répondre aux non-conformités soulevées lors de l'audit de certification (si non-conformités il y avait). Cette dernière étape de la certification a été réalisée dans un contexte difficile. L'entreprise connaît une réduction du volume d'affaires et recentre donc les priorités du personnel sur la productivité directe. Le temps consacré à la certification et à 
la consultation du personnel est réduit mais comme la plus grande partie de la formalisation des procédures internes demandées par la certification ont été réalisées en phase 2, cela n'handicape pas le processus de labellisation.

Cette phase est surtout marquée par un repositionnement d'un acteur du contexte externe, en l'occurrence PhonCall (nom fictif), le sous-traitant marocain, auquel plus de $40 \%$ de la production francophone est confiée. Après avoir approuvé volontairement son engagement sociétal et formalisé un engagement sur les pratiques qui comprenait un audit externe, PhonCall freine sa collaboration dans le processus de certification. Une consultante a été envoyée au Maroc avec comme objectif d'auditer la structure interne au regard des huit thèmes de la labellisation: liberté syndicale et de négociation collective, absence de travail des enfants, absence de discrimination et de travail forcé, santé et sécurité des travailleurs, temps de travail et heures supplémentaires. Pour rappel, les exigences de la norme SA 8000 pour certains de ces thèmes dépassent largement le droit social marocain. Quelques adaptations sont réalisées mais le dossier bloque sur deux aspects: les investissements nécessaires pour la mise en conformité avec les normes de sécurité des bâtiments mais aussi l'obligation d'avoir une représentation des travailleurs. Le directeur du centre d'appels ne souhaite pas qu'une délégation syndicale se mette en place; il argumente qu'un dialogue direct avec les travailleurs est plus opérationnel et préférable pour les parties en présence. On se retrouve donc à priori dans la même situation de blocage que dans la phase 2, toutefois à l'inverse de ce qui s'est passé dans l'étape précédente avec les grands fournisseurs de WebTel, celui-ci va pouvoir mobiliser sa position de donneur d'ordres pour convaincre PhonCall de monter un plan d'action pour s'ajuster progressivement aux exigences du label. WebTel est ici en position de pouvoir, lié à l'engagement organisationnel de cette partie prenante même si son engagement sociétal est faible (Sobzack et Girard, 2006). PhonCall a tout intérêt à maintenir son contrat de sous-traitance avec WebTel et la menace de perdre le marché s'il n'y a pas de mise en conformité ou du moins un plan d'action porte ses fruits.

En avril 2006, WebTel reçoit les auditeurs de certification sur ces deux sites d'exploitation de Wallonie et de Flandre. Elle doit prouver l'existence de procédures écrites formalisant la GRH, dont l'application est validée par les auditeurs par des entretiens avec les employés et la direction. Les procédures, les pratiques et les preuves des huit éléments de la norme sont minutieusement évaluées. Les procédures internes sont validées par les auditeurs avec une prise en compte de la réalité de PME qui n'oblige pas à une délégation syndicale et donc une assimilation à l'existence d'un délégué du personnel qui reste non protégé par la loi. 
Quant aux difficultés éprouvées lors de la mise en œuvre du processus de certification eu égard à certaines parties prenantes externes, WebTel arrive à justifier son impossibilité d'imposer aux grands fournisseurs un contrôle sur les pratiques. Elle apporte toutefois la preuve des démarches entreprises et les réponses apportées par ces fournisseurs, ainsi que les raisons qui expliquent pourquoi il lui serait trop coûteux de changer de fournisseur. Cela rejoint les travaux de Aggeri et al.(2005, p. 260) qui montrent que les entreprises ne sont pas démunies par rapport aux dynamiques de normalisation, qu'elles font partie du jeu et qu'elles s'engagent dans la négociation de ces normes.

En mai 2006, WebTel reçoit le label SA 8000. Il est intéressant de noter que ce sera la première PME européenne et le premier centre d'appels à l'obtenir. Pourtant, le directeur général refuse d'en faire un argument marketing pour deux raisons principales:

- la première réside dans le fait que l'objectif initial de la démarche était une mise en conformité par rapport à certaines exigences des donneurs d'ordres et une volonté de standardisation des procédures de contrôle sur tous les sites de production;

- la deuxième raison est qu'une action marketing de grande envergure risquait d'attirer l'attention des grands concurrents sur ce centre d'appels qui travaille un peu dans l'ombre des grands acteurs du secteur. WebTel n'a pas encore atteint la maturité pour pouvoir répondre à la demande du marché, en matière de taille, de ressources humaines et de capacité d'hébergement (travail le dimanche ou la nuit, par exemple). Une action marketing à cette période paraît inadéquate.

Les auditeurs ont accepté de labelliser l'entreprise alors qu'elle ne répondait pas totalement à tous les critères sur base de l'argumentation de l'impossibilité de WebTel d'aller plus loin compte tenu de ses moyens financiers et de son pouvoir interne et externe.

Cette étude de cas montre que le processus de labellisation est fortement marqué par des facteurs de contingence propres aux PME. Ainsi, la forte dépendance par rapport aux grands clients, donneurs d'ordres, va limiter la PME dans les étapes classiques de la certification. En effet, sa positon de dépendance et son faible pouvoir sur ces acteurs l'empêchent d'exiger un respect de toutes les dimensions du label, en l'occurrence un engagement et un contrôle sur les pratiques. Cela amène WebTel à opter pour une stratégie de contournement du processus de certification en faisant certifier le processus (sollicitation des parties prenantes) et pas les résultats. Elle aurait pu aller au-delà en menant une action qui viserait à convaincre les parties 
prenantes hésitantes de leur intérêt à se lancer dans le processus, mais ce processus risquait d'être long et complexe. Le peu de ressources qu'elle peut affecter à ce projet au vu de sa taille ne lui a pas laissé l'opportunité d'envisager cette possibilité.

L'analyse montre toutefois que la dimension politique est aussi importante. Ainsi, bien qu'elle ait peu de pouvoir sur certains de ces fournisseurs et sur les donneurs d'ordres, WebTel est par contre en position de pouvoir face à son sous-traitant, ce qui lui permet d'être plus exigeant à son égard sur la nécessité de se conformer aux exigences du label pour maintenir le partenariat commercial mis en place.

L'étude de cas fait aussi clairement ressortir le rôle clé que jouent les valeurs du dirigeant-propriétaire. Cela rejoint les constats de Ghobadian et Gallear (1996) et de Creplet et Lanoux (2000, p. 7) qui s'intéressent au rôle joué par le dirigeant dans l'adoption de systèmes qualité, fort proche de la démarche de labellisation. Le dirigeant doit en effet convaincre les parties prenantes de l'entreprise de la pertinence de la démarche et pour cela, «il doit lui-même être convaincu».

Notons enfin tout l'intérêt d'une analyse politique pour comprendre la façon dont le personnel va se positionner face aux opportunités qui se créent autour de ce processus. Le label va devenir un moyen pour obtenir une plus grande formalisation de la GRH et, donc, une réduction de l'arbitraire lié aux modes de gestion traditionnels des PME et la mise en place d'une délégation des travailleurs.

\section{Conclusion}

Notre objectif était de mener une analyse contextualiste du processus de labellisation SA 8000 d'une PME pour questionner le modèle rationaliste et universel généralement associé à ces processus de labellisation. Il s'agissait de voir si une PME pouvait se positionner au regard de ce label mais aussi d'établir les facteurs de contingence qui vont affecter le processus et l'impact des caractéristiques propres à une $\mathrm{PME}$.

L'analyse de mise en place du label SA 8000 comme outil de responsabilité sociale nous a permis de montrer qu'une restitution du processus sous un angle uniquement rationaliste, tel qu'il est présenté dans bon nombre de bonnes pratiques, est largement insuffisante pour comprendre le processus. Le processus de labellisation et le type de changement organisationnel qu'il génère sont largement dépendants des attributs des PME (effet de taille, pouvoir centralisé aux mains de l'équipe dirigeante) et de ses flux d'échange 
avec son environnement (position de dépendance par rapport aux donneurs d'ordres, pouvoir limité face aux fournisseurs puissants, position de pouvoir face à ses propres sous-traitants).

Les mécanismes à l'œuvre gagneraient à être validés par d'autres études de cas. Il reste un important travail d'analyse à réaliser autour du positionnement des parties prenantes en travaillant notamment sur le processus de mobilisation qui aurait pu être entamé pour convaincre les parties prenantes récalcitrantes à s'impliquer. Finalement, le texte met en évidence l'intérêt d'études qualitatives et longitudinales pour comprendre les processus de RSE dans les PME.

\section{Bibliographie}

Aggeri, F., E. Pezet, C. Abrassart et A. Acquier (2005), Organiser le développement durable. Expériences des entreprises pionnières et formation des règles de l'action collective, Paris, Vuibert.

Allouche, J. (2005), Corporate Social Responsibility, New York, Palgrave Macmillan.

Bayad, M., H. Mahé De Boislandelle, D. Nebenhaus et P. Sarnin (1995), «Paradoxe et spécificités des problématiques de gestion des ressources humaines en petites et moyennes entreprises », Gestion 2000, n 1, p. 95-108.

Bayad, M. et D. Nebenhaus (1998), «Contribution à un modèle pyramidal de la gestion des ressources humaines en PME», Revue internationale PME, vol.11, $\mathrm{n}^{\text {os }} 2-3, \mathrm{p} .161-178$.

Berger-Douce, S. (2004), «La diffusion du management environnemental en PME - Apports et limites d'une démarche collective», VII ${ }^{\mathrm{e}}$ Congrès international francophone en entrepreneuriat et PME, octobre, Montpellier.

Brouwers, I., A. Cornet et L. Gutierrez (1997), Management humain et contexte de changement, pour une approche constructiviste, Bruxelles, De Boeck.

Burns, T. et G.M. Stalker (1961), The Management of Innovation, Londres, Tavistock.

BusINESS AND SOCIETY (2005), «Premier baromètre de la responsabilité sociétale de l'entreprise en Belgique», <http://www.businessandsociety.be $>$.

Chaouki, F. et Z. Yanat (2004), «Contribution de la certification à l'amélioration de la compétitivité des entreprises marocaines: cas du secteur agro-alimentaire», Actes de la XXII ${ }^{e}$ Université d'été de l'Audit social, Luxembourg.

Creplet, F. et B. Lanoux (2000), Pour une approche dialogique du rôle de l'entrepreneur/ manager dans l'évolution des PME: l'ISO comme révélateur..., <http:// cournot2.u-strasbg.fr/users/beta/publications/2004/2004-03.pdf >.

Crozier, M. et E. Friedberg (1977), L'acteur et le système, Paris, Seuil, coll. «Sociologie politique».

Revue internationale P.M.E., vol. 20, nºs 3-4, 2007 
Dupuis J.-C., N. Haned et C. Le Bas (2006), «Les pratiques de RSE des PME, premières enquêtes auprès des PME de la Région Rhônes-Alpes », ESDES, $<$ http://www.esdes-recherche.net/PDF/RSEenquetelettre.pdf $>$.

Freeman, R.E. (1994), «The politics of stakeholder theory: some future directions », Business Ethics Quarterly, vol. 4, n 4, p. 409-422.

Ghobadian, A. et D.N. Gallear (1996), «Total quality management in SME's», Omega, vol. 24, n 1, p. 83-106.

Igalens, J. et J.M. Peretti (2004), «Contribution de l'audit social au respect des droits sociaux fondamentaux», Actes de la VII Université de printemps, Tunisie.

LAVAL, F. (1998), «La gestion des ressources humaines des entreprises fournisseurspartenaires: l'impact de la politique d'achat d'un grand groupe donneur d'ordres», Gestion des ressources humaines, vol. 11, $\mathrm{n}^{\text {os }} 2-3$.

LAWRENCE, P.R. et J.W. LoRSCH (1967), Adapter les structures de l'entreprise. Intégration ou différenciation, Paris, Éditions d'Organisation.

Mahé De Boislandelle, H. (1998), «GRH en PME. Universalité et contingences: essai de théorisation », Revue internationale PME, vol. 11, n ${ }^{\text {os }}$ 2-3, p. 11-29.

Miles, R.E. et C.C. Snow (1978), Organisational Strategy, Structure, and Process, New York, McGraw-Hill.

Mintzberg, H. (1982), Structure et dynamique des organisations, Paris/Montréal, Éditions d'Organisation.

Mitchell, R.K., B.R.Agle et D.J.Wood (1997), «Toward a theory of stakeholder identification and salience: defining the principle of who and what really counts », Academy of Management Review, vol. 22, $\mathrm{n}^{\circ}$ 4, p. 853-885.

Murillo, D. et J. Lozano (2006), «SMEs and CSR: an approach to CSR in their own words ", Journal of Business Ethics, vol. 67, n 3, p. 227-240.

ORSE (2005), «Le développement durable. Quels enjeux pour les PME?», <http:// orse.org/fr/home/news.html>.

Pettigrew, A.M. (1985), «Contextualist research: a natural way to link theory and practice», dans E.E. Lawler III (dir.), Doing Research that is Useful for Theory and Practice, San Francisco, Jossey Bass, p. 222-249.

Pettigrew,A.M. (1990), «Longitudinal field research on change: theory and practice», Organization Science, vol. 1, n 3, p. 267-292.

Pfeffer, J. (1981), Power in Organizations, Cambridge, Mass., Ballinger.

Pichault, F. (1993), Ressources humaines et changements stratégiques: vers un management politique, Bruxelles, De Boeck Université.

Pichault, F. et J. Nizet (2000), Les pratiques de gestion des ressources humaines: approche contingente et politique, Paris, Seuil.

Pichault, F. et M. Zune (2000), «Une figure de la déréglementation du marché du travail: le cas des centres d'appel», Management et conjoncture sociale, ${ }^{\circ} 580$, mai, p. 31-41. 
Quairel, C. et M.N. Auberger (2005), «Management responsable et PME: une relecture du concept de responsabilité sociétale de l'entreprise », dans J.-C. Dupuis et C. Le Bas (dir.), Le management responsable: vers un nouveau comportement des entreprises?, Paris, Economica, coll. «Gestion».

Sobzack, A. et C. Girard (2006), «Des parties prenantes aux parties prenantes engagées: pour une nouvelles lecture de la théorie des stakeholders », $\mathrm{I}^{\text {er }}$ Congrès du RIODD, Paris.

UNIONEUROPÉENNE (2006), «Introduction à la responsabilité sociale des entreprises dans les petites et moyennes entreprises », <http://ec.europa.eu/enterprise/csr/campaign/ documentation/download/introduction_fr.pdf $>$.

Revue internationale P.M.E., vol. 20, $\mathrm{n}^{\text {os }} 3-4,2007$

C 2008 - Presses de l'Université du Québec

Édifice Le Delta I, 2875, boul. Laurier, bureau 450, Québec, Québec G1V 2M2 • Tél.: (418) 657-4399 - www.puq.ca 